\title{
Optimum Luminescent Down-Shifting Properties for High Efficiency and Stable Perovskite Solar Cells
}

\author{
Miguel Alexandre*, Manuel Chapa, Sirazul Haque, Manuel J. Mendes*, \\ Hugo Águas, Elvira Fortunato, Rodrigo Martins \\ I3N/CENIMAT, Department of Materials Science, Faculty of Science and Technology, \\ Universidade NOVA de Lisboa and CEMOP/UNINOVA, Campus de Caparica, 2829-516 \\ Caparica, Portugal

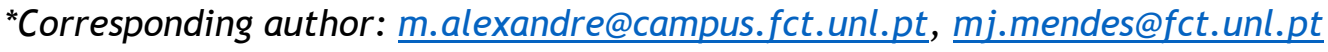

\begin{abstract}
In recent years, the discovery of the excellent optical and electrical properties of perovskite solar cells (PSCs) made them a main focus of research in photovoltaics, with efficiency records increasing astonishingly fast since their inception. However, problems associated with the stability of these devices are hindering their market application. UV degradation is one of the most severe issues, chiefly caused by $\mathrm{TiO}_{2}$ 's photo-generated electrons that decompose the perovskite absorber material, coupled with the additional intrinsic degradation of this material under UV exposure. The solution presented here can minimize this effect while boosting the cells' generated photocurrent, by making use of combined light-trapping and luminescent downshifting effects capable of changing the harmful UV radiation to higher wavelengths that do not affect the stability and can be effectively "trapped" in the cell. This work focuses in the optimization of the photocurrent gains that can be attained by emulating the changed spectrum resulting from applying down-shifting media as encapsulant in photonic-enhanced PSCs, as well as the reduction in the harmful effects of UV radiation on the devices. Such optimized photonic solution allows current enhancement while reducing the harmful UV photo-carrier generation both in the $\mathrm{TiO}_{2}$ (by one order of magnitude) and in the perovskite (by $80 \%$ ) relative to a standard PSC without light management.
\end{abstract}

Keywords: Photonics, Perovskite Solar Cells, Luminescent Down-shifting, UV Stability, Photocurrent Enhancement 


\section{Introduction}

The ever-growing cost-competitiveness of photovoltaic (PV) technology is the main reason behind the recent growth in total global PV installed capacity (increasing from under $50 \mathrm{GW}$ to above $400 \mathrm{GW}$ from 2010 to 2017). ${ }^{1-3}$ Crystalline silicon (c-Si) solar cells have been the uncontested market leaders of this technology. 2,4 However, the need to keep fueling this growth has turned the researchers' attention to other materials and light management techniques, as the sub-optimal absorption of c-Si is an obstacle in a scenario of ever-decreasing material thickness, ${ }^{5,6}$ which is a fundamental factor for low cost and flexible photovoltaics. ${ }^{7,8}$ Therefore, novel materials with excellent optical properties have been studied to achieve lower thickness without imperiling the device's performance. The class of materials that, in recent years, has stood out from the rest are hybrid organic-inorganic perovskites (HOIPs), as they exhibit direct bandgap, ${ }^{9-11}$ long carrier diffusion lengths, ${ }^{12,13}$ and high optical absorption coefficient. ${ }^{11,12,14}$ The evidenced properties paved the way for the fast adoption of these materials, thus leading to a steep increase in PV conversion efficiency reaching values above $22 \%$ in just a few years. ${ }^{9,13,15-17}$ HOIPs are based on an $\mathrm{ABX}_{3}$ atomic structure, where $\mathrm{A}$ and $\mathrm{B}$ are cations of different size and $\mathrm{X}$ is an anion. ${ }^{9,18}$ The first is an organic or inorganic ion (methylammonium, $\mathrm{CH}_{3} \mathrm{NH}_{3}{ }^{+}$; ethylammonium, $\mathrm{CH}_{3} \mathrm{CH}_{2} \mathrm{NH}_{3}{ }^{+}$; formamidinium, $\mathrm{NH}_{2} \mathrm{CH}=\mathrm{NH}_{2}{ }^{+}$, $\mathrm{Cs}$ and $\mathrm{Rb}$ ), the second a divalent metal cation $\left(\mathrm{Ge}^{2+}, \mathrm{Sn}^{2+}\right.$ and $\left.\mathrm{Pb}^{2+}\right)$. The last element on the structure, $\mathrm{X}$, is a monovalent halogen anion $\left(\mathrm{F}^{-}, \mathrm{Cl}^{-}, \mathrm{Br}^{-}, \mathrm{I}^{-}\right) .9,12,18$

Although perovskite solar cells (PSCs) display an impressive performance, competing with the best silicon solar cells in terms of efficiency, they have several stability problems mainly related with exposure to moisture, oxygen and UV radiation, that are responsible for blocking its market implementation. ${ }^{18}$ These degradation mechanisms are not yet fully understood, however several studies have defined many possible mechanisms. ${ }^{19-21}$ Moisture and oxygen exposure can be mostly prevented via proper encapsulation, ${ }^{21}$ leaving UV exposure as the most important susceptibility to overcome. The UV degradation of PSCs has been attributed to photocatalytic effects caused by $\mathrm{TiO}_{2}$, the material commonly used as electron transport layer (ETL). This effect was first reported by Leijtens et. al. where it was hypothesized that, upon electron excitation in $\mathrm{TiO}_{2}$, deep trap states are created that capture photo-electrons generated in the absorber material, therefore hindering the cell's performance. ${ }^{22}$ Ito et. al. also suggested that the formation of $\mathrm{I}_{2}$, due to electron transfer to deep trap states in the $\mathrm{TiO}_{2}$ /perovskite interface, can then decompose the perovskite crystal by evaporation of volatile compounds. ${ }^{20}$ Regarding perovskite 
degradation, excluding the $\mathrm{TiO}_{2}$ 's effects, Quistch et. al. calculated the threshold energy between the perovskite's photo-brightening and photo-degradation, correlating it with the presence of residual $\mathrm{PbI}_{2}$. This study indicated that $\mathrm{I}_{2}$ can be created by the photolysis of $\mathrm{PbI}_{2}$, leading to an equivalent degradation process to the aforementioned one. ${ }^{23}$

Considering the above-mentioned problems, several techniques have been used to mitigate these degradation mechanisms, such as replacing $\mathrm{TiO}_{2}$ by other nonharmful materials, ${ }^{22}$ depositing blocking layers between the $\mathrm{TiO}_{2} /$ perovskite interface to avoid electron exchange, ${ }^{20}$ as well as the use of either a UV filter or a down-shifting (DS) layer that prevents UV radiation from reaching the cell. ${ }^{22,24-26}$ This latter case is the one that was unprecedentedly optimized in this study.

Down-shifting is the process where high energy photons are converted to lower energy photons. ${ }^{27,28}$ The main materials used for this process are quantum dots (QDs), ${ }^{29-32}$ dyes ${ }^{33-35}$ and rare-earth elements. ${ }^{36-38}$ These materials have recently been applied to perovskite, ${ }^{25,26}$ organic $^{39}$ and dye sensitized solar cells ${ }^{40}$ to improve their life-time without compromising their overall performance. When compared with the use of UV filters, which is the current common way of enhancing the UV stability of PSCs, ${ }^{22}$ using DS benefits from the exploitation of the energy of otherwise lost photons, while still protecting the absorber material from the harmful UV radiation. One example, by Anizelli et. al., compared the results against a UV filter obtaining a similar development of the device's parameters with prolonged UV exposure. ${ }^{25}$

As stated earlier, the need for ever-decreasing thickness without compromising solar cell performance is a driver for research in the PV field. However, decreasing thickness leads to lower red-NIR absorption, as this radiation has higher penetration depth. Therefore, different light-trapping (LT) methods have been studied to improve this low-energy absorption, such as the use of metallic or dielectric nanoparticles, ${ }^{41-}$ 43 front or rear texturing of the cell ${ }^{6,44,45}$ and the use of high index dielectric structures on the cell front. ${ }^{7,46,47}$ This latter LT method, operating in the regime of wave-optics, is the one considered in this work. Front-located high index photonic structures allow strong forward scattering of light, thus increasing its travel path within the PV absorber, as well as the creation of resonant modes, related with the structure's properties, that can greatly boost the cell's absorption. For periodic structures, this increase can even surpass the theoretical limit - the Tiedje-Yablonovitch limit - for specific wavelengths related with the LT structure's pitch. $6,47,48$ 


\section{Results and discussion}

In this study, two different types of solar cells were considered (Figure 1 a) and b)): First, a planar cell, henceforth PC, and a cell with light trapping structures, henceforth LTC. The absorber material considered in the PSCs is the conventional best-performing $\mathrm{CH}_{3} \mathrm{NH}_{3} \mathrm{PbI}_{3}\left(\mathrm{MAPbI}_{3}\right)$ perovskite. ${ }^{12,18}$ Two different thickness values for the perovskite layer were used, namely 250 and $500 \mathrm{~nm}$, as the latter value is that conventionally used in PSCs while the former is of interest for the development of ultra-thin photonic-enhanced devices. $8,9,16,49$ The remaining solar cell dimensions and materials are based on previous results from optimizations centered on maximizing the optical performance of front LT structures on the PSCs, ${ }^{49}$ which are summarized in Table S1 of Supplementary Material. A preferential implementation of luminescent down-shifting (LDS) materials is via their embedment in a polymeric matrix used as the cell's encapsulant, ${ }^{24,25,50}$ as sketched in Figure $1 \mathrm{a}$ ) and b), which is generally several micrometers thick. ${ }^{24,50}$ Therefore, such DS encapsulant layer was modelled as the background medium over the cells' structures. This way, in the simulation, the background refractive index ( $n_{\text {Background) }}$ was set to 1.5 as it is a common value for transparent polymeric materials used for this purpose such as PMMA, ${ }^{25}$ EVA, ${ }^{50}$ PVB ${ }^{50}$ and PS. ${ }^{24}$ A base simulation with vacuum background index ( $n_{B a c k g r o u n d}=1$ ) was still made for the PCs, for comparison.

The present work shows how to ideally design the DS effect in order to attain: 1) maximum optical improvement in the PSCs, represented in terms of their overall photocurrent density, $\mathrm{J}_{\mathrm{ph}}$; 2) negligible UV degradation by minimizing both the light absorption in the $\mathrm{TiO}_{2}$ across the full spectral range, and the UV photocurrent (i.e. carrier generation in the perovskite due to UV absorption) calculated for wavelengths between 300 and $400 \mathrm{~nm}$, henceforth Jph-uv.

The results were obtained using a numerical solver (FDTD Solutions) provided by Lumerical Inc. ${ }^{51}$ The use of this method allows for the calculation of the electric and magnetic fields in the entire simulation region. Based on the fields' values, the absorbed power density can be determined by: $A b s=-0.5 \omega|E|^{2} \operatorname{imag}(\epsilon)$, where $\omega$ is the angular frequency, $\epsilon$ is the material permittivity and $E$ is the electric field at a given point in the simulation region. By integrating this expression in volume, it is possible to calculate the cell's absorption spectrum, $A(\omega)$. Then, together with the solar spectrum incident on the cell, one can calculate $\mathrm{J}_{\mathrm{ph}}$ using: $J_{p h}=\int A(\omega) A M_{1.5 G} d \omega$, where $\mathrm{AM}_{1.5 \mathrm{G}}$ is the spectrum incident on the cell. The complex refractive index spectra of the materials considered in this study are plotted in Figure S1. The accuracy of the model was corroborated by extended validation tests described in Section S3. 
a) Planar Cell (PC)

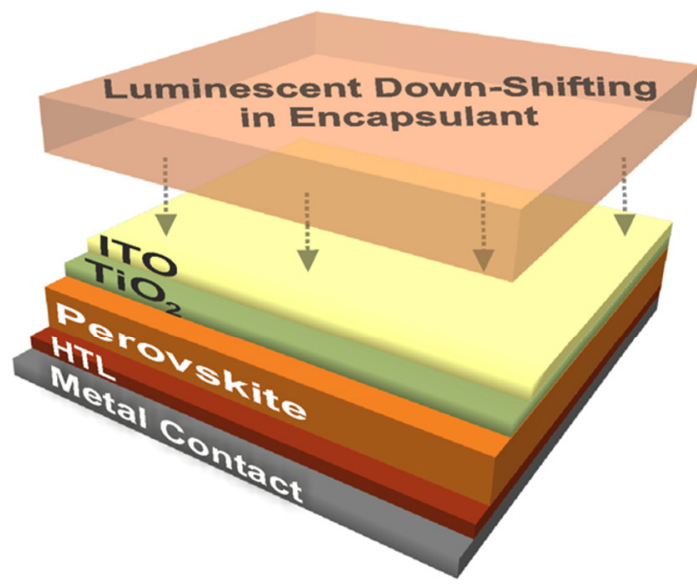

b) Light Trapping Cell (LTC)

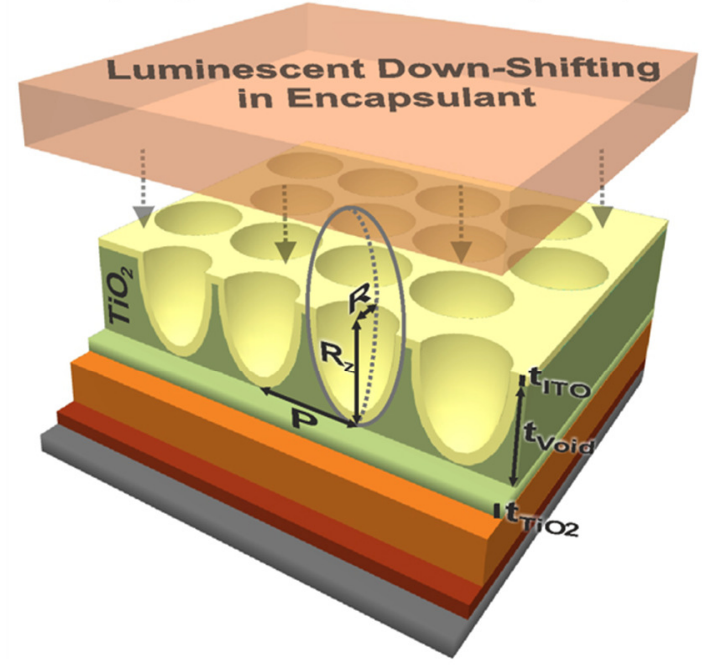

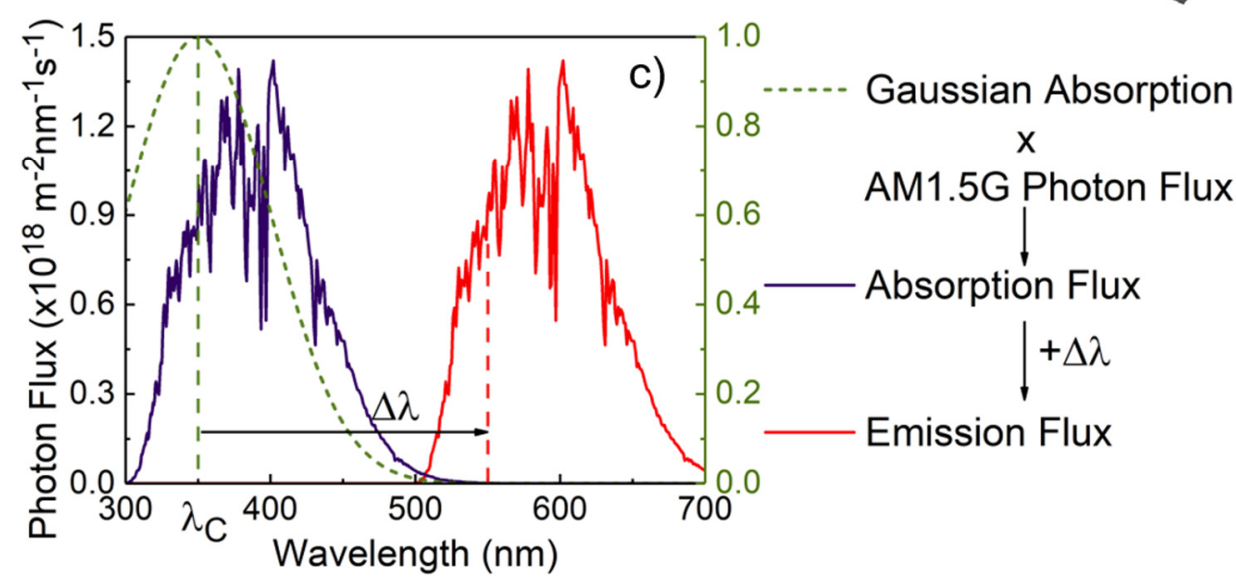

d)

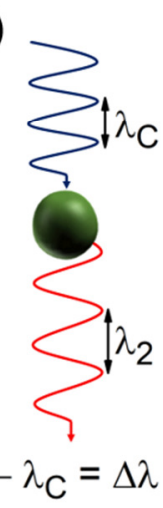

Figure 1 - Schematic of the solar cell structures considered in the simulations. a) Planar cell structure used as reference, composed by the layers: Metal contact (Ag)/Hole Transport Layer (HTL, made of Spiro-OMeTAD)/Absorber (Perovskite)/ Electron Transport Layer (ETL, made of $\mathrm{TiO}_{2}$ ), Transparent conductive oxide (TCO, made of ITO). b) Light trapping cell structures integrated in the n-contact (ETL) of the PSC. The corresponding cell dimensions are shown in Supplementary Material - Table S1. c) Plot illustrating the method used to emulate the process of down-shifting. In dashed green is the gaussian profile, in blue is the absorbed flux and in red is the emitted flux; d) schematic depicting the process of down-shifting by an LDS material.

Since this is an electromagnetic method based on Maxwell's equations, it cannot exactly simulate the effect of DS. Consequently, here DS was modelled by proper adaptation of the incident spectrum, as described in Figure $1 \mathrm{c}$ ). Based on absorption and emission profiles of typical LDS materials, taken from several reports, ${ }^{25,33,35,39,52-}$ 54 a gaussian profile was chosen to emulate these properties - Figure $1 \mathrm{c}$ ) green dashed profile. Gaussian profiles have three main variables: the gaussian RMS width, parameter related with the FWHM which was fixed at $50 \mathrm{~nm}$; the gaussian center, $\lambda_{\mathrm{C}}$, that was left as a variable; and the amplitude, representing the peak absorption, that was fixed at unity. This gaussian absorption was then multiplied with the solar photon 
flux, based on the ASTM G-173 global irradiance spectra provided by NREL, ${ }^{55}$ to calculate the hypothetical absorption flux in a thick DS layer - Figure $1 \mathrm{c}$ ) blue profile. Subsequently, this absorbed flux is shifted to higher wavelengths, as shown in Figure $1 \mathrm{c})$, by a shifting parameter, $\Delta \lambda$, that was also left as a variable. Lastly, to create the "shifted" spectrum incident on the PSC, the absorbed flux was subtracted, while the emission flux was added to the pristine AM1.5G spectrum. As an example, Figure S2 shows the resulting spectral irradiance plots for two different $\Delta \lambda$ and $\lambda_{c}$. It should be noted that the process used to emulate the DS process is an ideal one and, thus, does not account for effects such as isotropic emission, non-unitary quantum efficiency and reabsorption.

Firstly, the solar cells' absorption profiles were determined by the aforementioned method and are displayed in Figure $2 \mathrm{a}$ ) and b) for the PCs and in Figure $3 \mathrm{a}$ ) and b) for the LTCs, considering the $n_{\text {Background }}=1.5$. The profiles for $n_{\text {Background }}=1.0$ are shown in Section S4 of Supplementary Material. These absorption profiles only depend on the solar cells' structure and materials, being independent of the illumination spectrum. Therefore, they are suited to evaluate the device's optical performance. For red-NIR wavelengths there is a decrease in the cell absorption as sub-bandgap photons are harder to absorb. This is effectively shown by the absorbed power density plots shown for $900 \mathrm{~nm}$ wavelength (rightmost inset profiles in Figure 2 and Figure 3), as the absorption is rather uniform throughout the cell. Note that, for the LTCs, these losses are reduced by the use of the photonic structures responsible for scattering light. For shorter wavelengths, the most notable aspect is the parasitic absorption from $\mathrm{TiO}_{2}$ and ITO for both PCs and LTCs. The absorbed power density plots (leftmost inset plots in Figure 2 and Figure 3 at $350 \mathrm{~nm}$ wavelength) again serve to further verify this statement, as they show a pronounced absorption in the $\mathrm{TiO}_{2}$ and ITO layers. Furthermore, the LTCs show an effective UV shading of the perovskite, revealed by the red shaded area in the absorption spectra of Figure 3 a) and b) as well as the substantial absorbed power density in the front LT structures shown by the $350 \mathrm{~nm}$ inset profiles in Figure 3. This increase is due to the thick $\mathrm{TiO}_{2}$ used in these structures, as it is the material responsible for the light trapping effects (i.e. improved broadband anti-reflection and scattering). ${ }^{49}$ It should be noted that such UV shading is also beneficial for the perovskite cells, due to the UV instability problems.

Subsequently, sweeps were made for the different cells in study, where $\Delta \lambda$ and $\lambda_{c}$ where varied between $100-400 \mathrm{~nm}$ and $300-400 \mathrm{~nm}$, respectively. The resulting photocurrent contour plots for the PCs are shown in Figure $2 \mathrm{c}$ ) and d), and for the LTCs in Figure $3 \mathrm{c}$ ) and d). The equivalent results for the PCs with $\mathrm{n}_{\text {Background }}=1$ are shown in Section S5. 


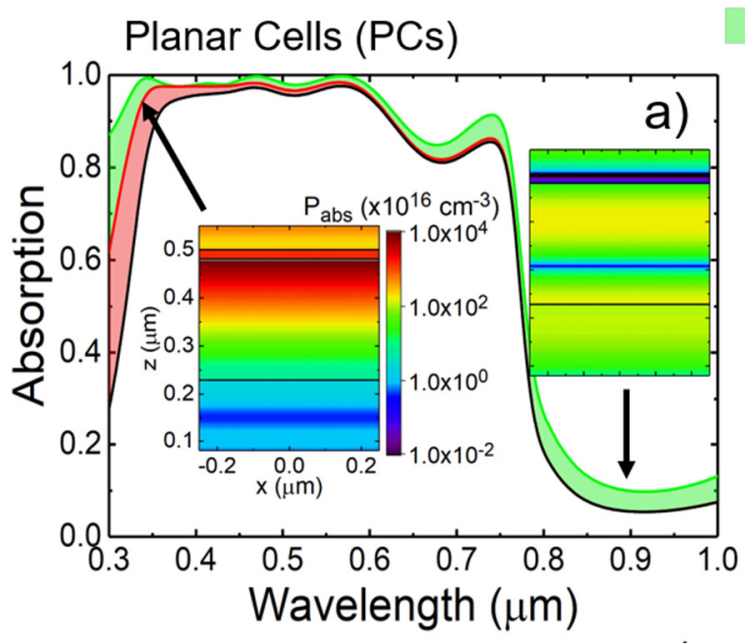

Jsc

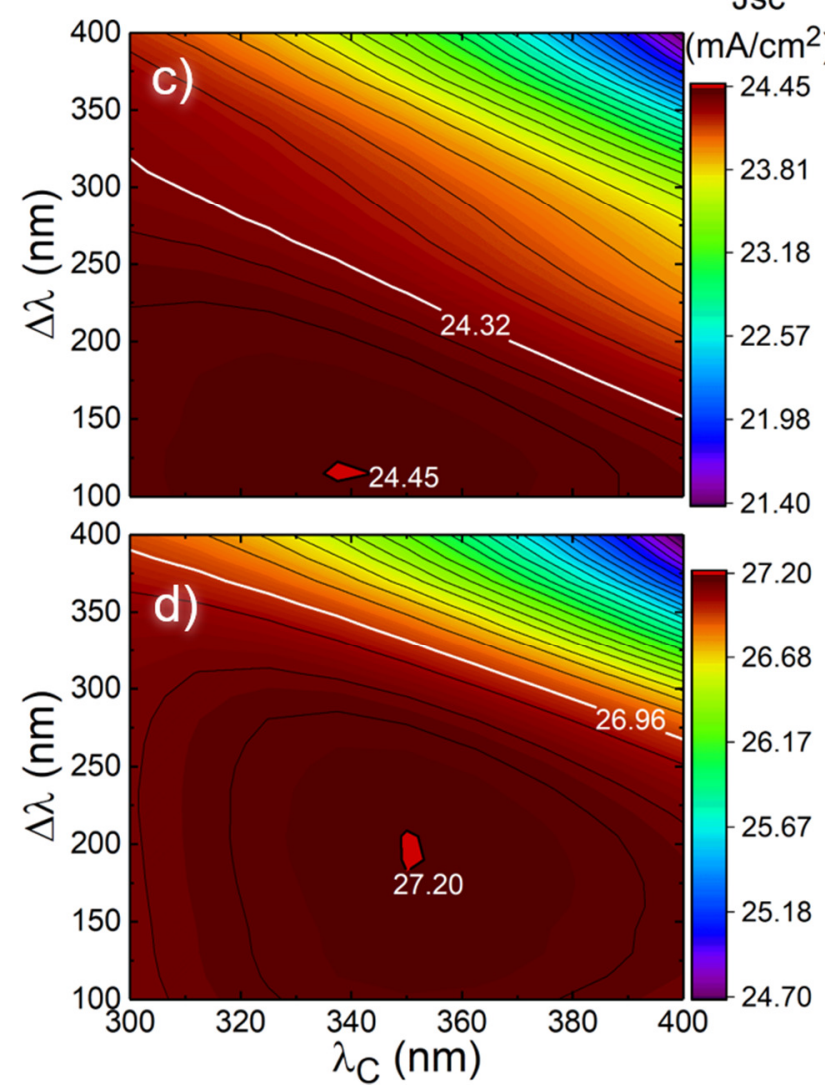

Total Perovskite $+\mathrm{TiO}_{2}-$ Perovskite

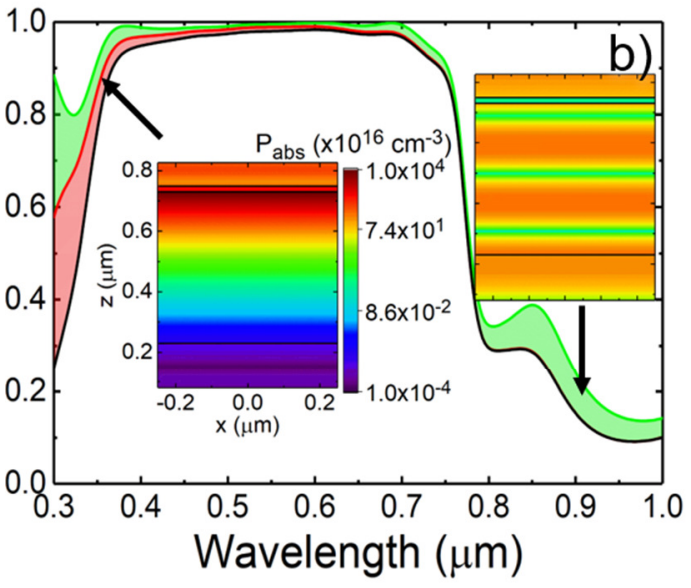

Photo-generation $\left(\times 10^{26} \mathrm{~cm}^{-3} \mathrm{~s}^{-1}\right)$
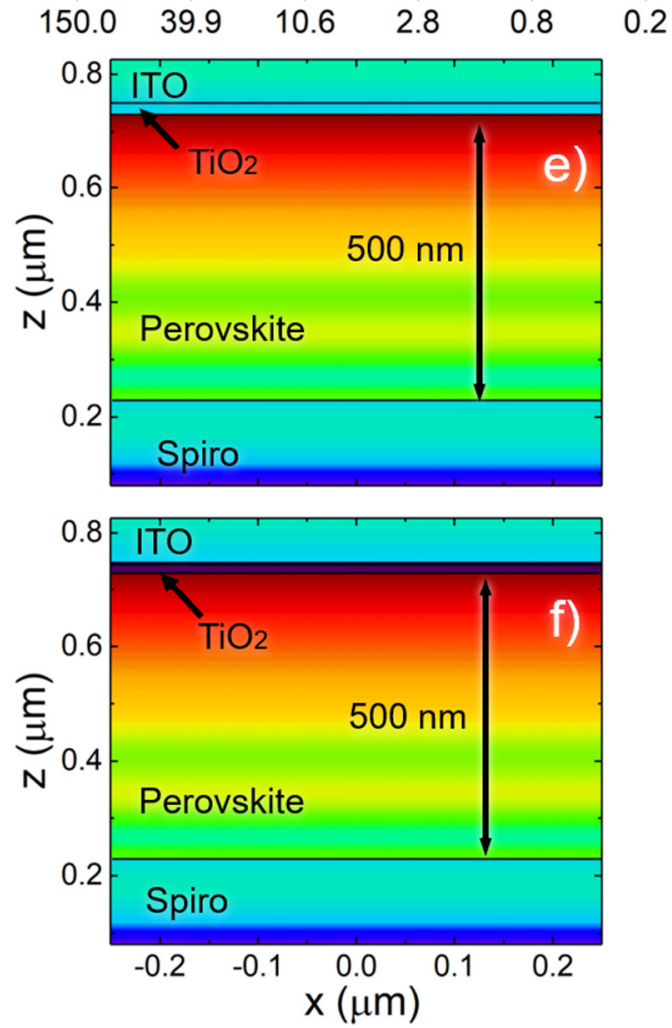

Figure 2 - Results for the PCs structure with $n_{\text {Background }}$ of 1.5. a) and b) are the absorption profiles calculated for the PCs with perovskite thickness of 250 and $500 \mathrm{~nm}$, respectively. The black curve corresponds to the light absorption in the perovskite layer; in red, the perovskite $+\mathrm{TiO}_{2}$ absorption; and, in green, the total absorption of the cell. The inset plots represent the absorbed power density through the cross section of the cell, calculated at specific wavelengths indicated by the arrows: the leftmost profile is the absorbed power for $350 \mathrm{~nm}$ and the rightmost graph is the absorbed power for $900 \mathrm{~nm}$; c) and d) are the contour plots of the photocurrent density $\left(\mathrm{J}_{\mathrm{ph}}\right)$ sweeps performed for the PC structure with $250 \mathrm{~nm}$ and $500 \mathrm{~nm}$ perovskite layer, respectively; the white contour line represents the pristine $J_{\text {ph, }}$ i.e. the value attained with the AM1.5 incidence spectrum without any shifting. e) and f) are examples of the carrier generation profiles for the $P C$ with $500 \mathrm{~nm}$ perovskite layer, considering the pristine and the optimized (using the $\lambda_{c}$ and $\Delta \lambda$ corresponding to the maximum in $d$ )) incidence spectrum, respectively. 

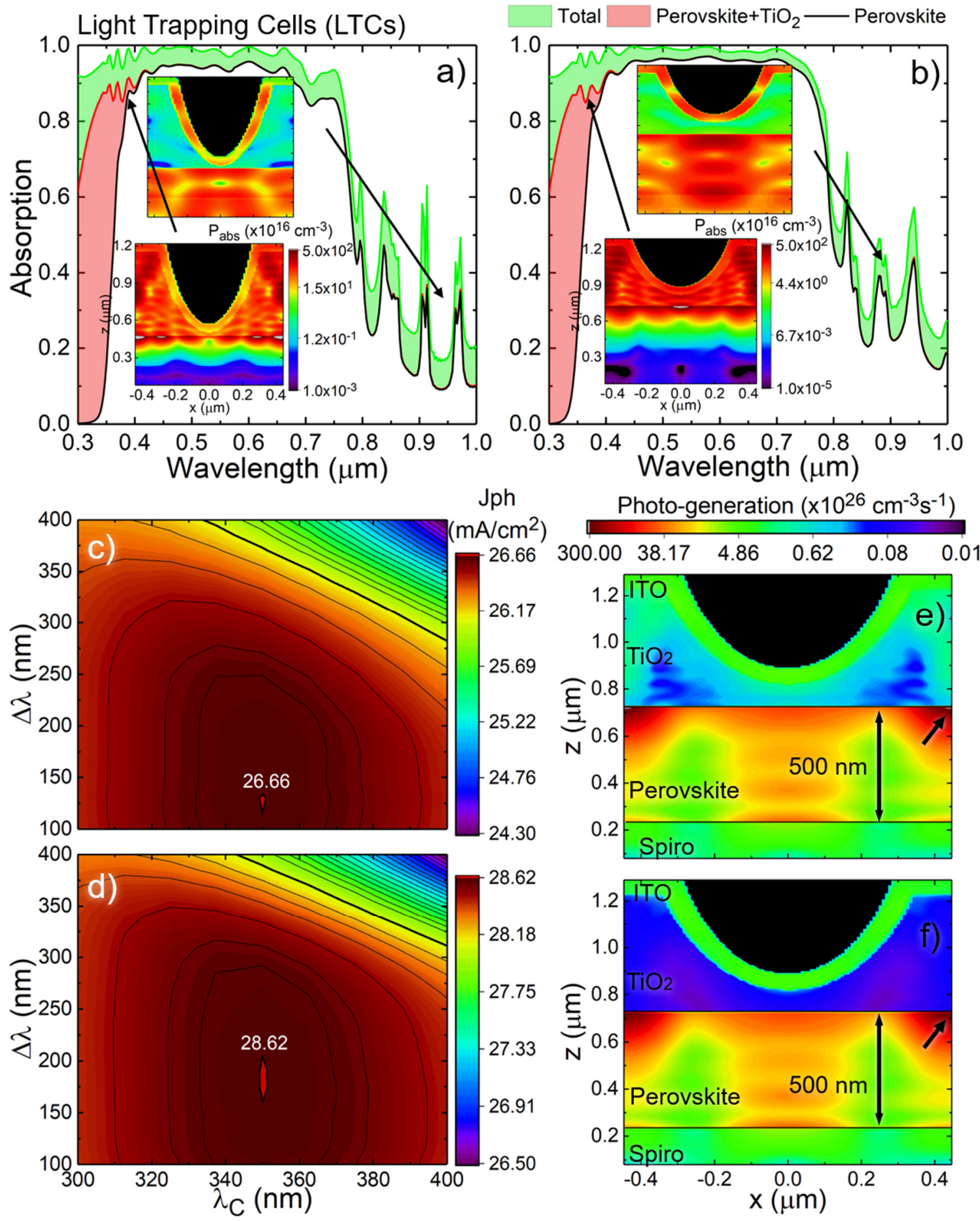

Figure 3 - Results for the LTCS with $n_{\text {Background }}$ of 1.5. a) and b) are the absorption profiles calculated for the LTCs perovskite thickness of 250 and $500 \mathrm{~nm}$, respectively. The black curve corresponds to the light absorption in the perovskite layer; in red, the perovskite $+\mathrm{TiO}_{2}$ absorption; and, in green, the total absorption of the cell. The inset plots represent the absorbed power density through the cross section of the cell, calculated at specific wavelengths indicated by the arrows: the leftmost profile is the absorbed power for $350 \mathrm{~nm}$ and the rightmost graphs is the absorbed power for $900 \mathrm{~nm}$. c) and d) are the contour plots of the $J_{p h}$ sweeps performed for the LTC structure with $250 \mathrm{~nm}$ and $500 \mathrm{~nm}$ perovskite layer, respectively; the thicker contour line represents the pristine $J_{p h}$, i.e. the value attained with the AM1.5 incidence spectrum without any shifting. e) and f) are examples of the carrier generation profiles for the LTC with $500 \mathrm{~nm}$ perovskite layer, considering the pristine and the optimized (using the $\lambda_{c}$ and $\Delta \lambda$ corresponding to the maximum in $d$ )) incidence spectrum, respectively. 
Firstly, one should note that the photocurrent contour plots (in Figure 2 and Figure 3) show a similar behavior, which is expected as these results are chiefly influenced by the absorption in the Perovskite layer, which is mostly similar in the 400-700 $\mathrm{nm}$ wavelength range for the different solar cells (Figure $2 \mathrm{a}$ ) and b) and Figure $3 \mathrm{a}$ ) and b)). At around $700 \mathrm{~nm}$, value close to the perovskite's bandgap, ${ }^{10,14}$ a significant drop in absorption occurs, as below-bandgap absorption is significantly reduced. Thus, it is expected that the photocurrent sweeps should also see this effect when the shifting occurs to higher wavelengths. Indeed this is the case, and, for the LTCs, this drop can be up to $7 \%$ lower than that of the corresponding pristine (without DS) structure (shown in Figure $3 \mathrm{c}$ ) and d) by the white line), while for the PC this value can be up to $12 \%$ lower. Therefore, it should be noted that the use of unoptimized LDS material properties can severely degrade the solar cell's performance.

Considering the photocurrent gains summarized in Table 1, when comparing the pristine AM1.5G spectrum with the shifted spectra, one can see that there is only a small increase in the cell's performance, even when considering the optimized $\lambda_{C}$ and $\Delta \lambda$ values indicated in Table 1 . For the LTCs, this $J_{p h}$ increase was up to $\sim 2 \%(\sim 0.6$ $\left.\mathrm{mA} / \mathrm{cm}^{2}\right)$, while for the PCs it was up to $\sim 1 \%\left(0.2-0.3 \mathrm{~mA} / \mathrm{cm}^{2}\right)$, with the main difference being attributed to higher $\mathrm{TiO}_{2}$ thickness in the first, as this layer is mainly responsible for UV parasitic absorption. It should be noted that, when integrating the ASTM G-173 solar irradiance spectra in the UV wavelength range (i.e. from 300-400 $\mathrm{nm}$ ), the maximum current density that can ideally be generated is only $\sim 1.4$ $\mathrm{mA} / \mathrm{cm}^{2}$, which sets the limit for the increase in photocurrent that can be attained using DS methods. Secondly, the outstanding perovskite's absorption properties are also a factor limiting further photocurrent increases. From the absorption profiles shown in Figure 2 and Figure 3, it can be seen that under $400 \mathrm{~nm}$ there is still some significant perovskite absorption. As such, and understandably, LDS materials cannot directly provide major photocurrent improvements in PSCs.

Nevertheless, it should be emphasized that the main objective of this method is to reduce the harmful effects of UV radiation on PSCs, and for that the optimized DS+LT solution presented here is shown to be outstanding. On the other hand, it should be noted that, even though electrical effects were not taken into account in this work, the enhancement in the overall opto-electronic performance (i.e. conversion efficiency) of the devices may surpass the photocurrent gains presented here. This is due to the fact that the sum of the optimized $\Delta \lambda$ and $\lambda_{C}$ values (resulting in the wavelength to where the shifting occurs) nears $500 \mathrm{~nm}$, which is the spectral position of the peak of the external quantum efficiency of this type of PSCs, as determined in previous contributions. ${ }^{16,25,26,56}$ 
Table 1 - Summary of the main results from the photocurrent density sweeps performed in this work. tPerovskite represents the perovskite thickness, $n_{\text {Background }}$ is the refractive index used for the background medium, pristine $J_{p h}$ is the value using the illumination spectrum without any shifting, optimized $J_{p h}$ is the highest value obtained in the sweeps of Figure 2 and Figure $3, \lambda c$ and $\Delta \lambda$ is the gaussian center and shifting parameter, respectively, corresponding to the maximum photocurrent value obtained in the sweeps.

\begin{tabular}{|c|c|c|c|c|c|c|}
\hline \multirow{3}{*}{$\begin{array}{c}n_{\text {Background }} \\
\text { tPerovskite }_{(\mathrm{nm})}\end{array}$} & \multicolumn{4}{|c|}{ Planar Cell (PC) } & \multirow{2}{*}{\multicolumn{2}{|c|}{$\begin{array}{c}1.5 \\
1.5\end{array}$}} \\
\hline & \multicolumn{2}{|c|}{1} & \multicolumn{2}{|c|}{1.5} & & \\
\hline & 250 & 500 & 250 & 500 & 250 & 500 \\
\hline Pristine $\mathrm{J}_{\mathrm{ph}}\left(\mathrm{mA} / \mathrm{cm}^{2}\right)$ & 22.9 & 25.9 & 24.3 & 27.0 & 26.1 & 28.1 \\
\hline Optimized $\mathrm{J}_{\mathrm{ph}}\left(\mathrm{mA} / \mathrm{cm}^{2}\right)$ & 23.0 & 26.3 & 24.5 & 27.2 & 26.7 & 28.6 \\
\hline$\lambda_{c}(\mathrm{~nm})$ & 387.5 & 400.0 & 337.5 & 350.0 & 350 & 350 \\
\hline$\Delta \lambda(\mathrm{nm})$ & 145 & 205 & 115 & 205 & 130 & 190 \\
\hline
\end{tabular}

Afterwards, the generation profiles were calculated using the optimum shifting parameters $(\lambda c, \Delta \lambda)$ obtained from the photocurrent sweeps (Table 1$)$. These profiles are shown in Figure $2 \mathrm{e}$ ) and f) for the PC and Figure $3 \mathrm{e}$ ) and f) for the LTC, considering $500 \mathrm{~nm}$ perovskite thickness. The remaining generation profiles for the PCs and LTCs with $250 \mathrm{~nm}$ perovskite thickness are presented in Section S6. In all these cases, a major reduction in the $\mathrm{TiO}_{2}$ 's photo-generation is observed resulting from the use of a shifted spectrum. Examining the LTC case (Figure 3 e) and f)), it can be seen that these values vary from around $1.6-0.2 \times 10^{26} \mathrm{~cm}^{-3} \mathrm{~s}^{-1}$ for the pristine spectrum to $0.1-0.02 \times 10^{26} \mathrm{~cm}^{-3} \mathrm{~s}^{-1}$ for the optimized spectrum, representing a pronounced change of one order of magnitude. Therefore, optimally-shifting the spectrum can almost eliminate the $\mathrm{TiO}_{2}$ 's harmful photo-generation and, in practice, lead to improved device stability. On the other hand, when comparing Figure 3 e) and $\mathrm{f}$ ) it can also be seen a reduction in the absorption in the perovskite in the reddish "corner" region indicated by the arrow. This stems from the higher wavelength radiation incident upon the cell, that has a higher penetration depth, resulting in a higher solar cell bulk absorption instead of front surface absorption. A similar effect is also seen in Figure $2 \mathrm{f}$ ), where the bulk generation is higher from this effect. Electrically, this shift from surface to bulk absorption can also have a beneficial impact in the solar cell performance, as in surface absorption there is more electron recombination, particularly in PSCs. ${ }^{57}$

Thereupon, the photocurrent values for wavelengths ranging from 300-400 nm ( $\mathrm{J}_{\text {ph-Uv }}$ representing the UV absorption) were calculated using the pristine and optimized spectra, in order to assess how the shifting can impact the perovskite's UV absorption. These values are summarized in Table 2. Taking the example of the LTC with $250 \mathrm{~nm}$ perovskite thickness, for the pristine spectrum the calculated $\mathrm{J}_{\mathrm{ph}-\mathrm{uv}}$ was 
$0.7 \mathrm{~mA} / \mathrm{cm}^{2}$, while for the optimized spectrum this value was $0.1 \mathrm{~mA} / \mathrm{cm}^{2}$, representing a reduction of $86 \%$ in the harmful UV photo-generation in the perovskite. The reductions for the other cases are all also around 80\% (Figure $4 \mathrm{~b}$ )). Therefore, a remarkable reduction in the perovskite's UV absorption is determined as a result of the optimized shifting. Consequently, both the reduction in the perovskite's UV absorption and the lower $\mathrm{TiO}_{2}$ 's harmful photo-generation, are expected to yield a pronounced improvement in the life-time of PSCs implementing such DS solution. From the generation profiles for the LTC (Figure 3 e) and f)), it can be seen that the photo-generation in the $\mathrm{TiO}_{2}$ close to the perovskite interface (a critical region for the degradation mechanisms to occur) ${ }^{20,22}$ is lower than at the topmost part of the $\mathrm{TiO}_{2}$ structures. This reduction occurs due to the UV shading effect that comes from the use of relatively-thick high index front structures, providing further protection against UV radiation for the device.

Table 2 - Summary of the photocurrent density values, $J_{p h-U V,}$ calculated only in the UV wavelength range $(300-400 \mathrm{~nm})$ for the perovskite layer with the pristine, i.e. the unaltered $A M_{1.5}$ spectrum, and the optimized spectrum, i.e. the spectrum using the optimized downshifting parameters obtained in the photocurrent sweeps of Figure 2 and Figure 3.

\begin{tabular}{|c|c|c|c|c|c|c|}
\hline & \multicolumn{4}{|c|}{ Planar Cell (PC) } & \multirow{2}{*}{\multicolumn{2}{|c|}{$\begin{array}{c}\text { Lit Cell (LTC) } \\
1.5\end{array}$}} \\
\hline \multirow{2}{*}{$\begin{array}{c}\text { n Background } \\
\text { tPerovskite }(\mathbf{n m})\end{array}$} & \multicolumn{2}{|c|}{1} & \multicolumn{2}{|c|}{1.5} & & \\
\hline & 250 & 500 & 250 & 500 & 250 & 500 \\
\hline Pristine $\mathrm{J}_{\mathrm{ph}-\mathrm{uv}}\left(\mathrm{mA} / \mathrm{cm}^{2}\right)$ & 1.0 & 1.0 & 1.2 & 1.1 & 0.7 & 0.7 \\
\hline Optimized $\mathrm{J}_{\mathrm{ph}-\mathrm{uv}}\left(\mathrm{mA} / \mathrm{cm}^{2}\right)$ & 0.2 & 0.2 & 0.3 & 0.2 & 0.1 & 0.1 \\
\hline
\end{tabular}

The bar chart of Figure 4 summarizes the key results from this work. Starting with the photocurrent chart (Figure 4 a)), a first point to be made is the higher current for the $500 \mathrm{~nm}$ PSCs, resulting from the thicker absorber. This difference is due to higher red-NIR radiation absorption, as demonstrated in Figure 2 b) for the PC and Figure $3 \mathrm{~b}$ ) for the LTC. Secondly, the higher $\mathrm{J}_{\mathrm{ph}}$ for the PC with nBackground of 1.5 , when compared to the equivalent cell with index of 1 , stems from better index matching between the background and the front material in the cell (ITO), as seen in Figure S3. On the other hand, it can also be seen that the LTCs have higher $\mathrm{Jph}_{\mathrm{ph}}$ when compared with their planar counterpart. This increase clearly demonstrates the benefits of using photonic structures for LT, allowing for optically thick but physically thin devices.

The graph also indicates small current increases when using the optimized spectrum. Considering that electrical losses are neglected in these studies, it can be inferred from Figure 4 a) that these materials do not show a pronounced increase in optical performance. However, these same effects, such as bulk instead of surface 
absorption and lower thermal losses that come with higher energy transitions, can be deciding factors leading to improved cell current.

The bar chart analyzing the $\mathrm{J}_{\mathrm{ph}-\mathrm{uv}}$ in Figure $4 \mathrm{~b}$ ) shows that a remarkable reduction of around $80 \%$ is observed for all cases in the UV light absorption by the perovskite material. Therefore, by maintaining a similar optical current, while significantly reducing the harmful effects of UV radiation in these cells, an increased long-term performance is anticipated.
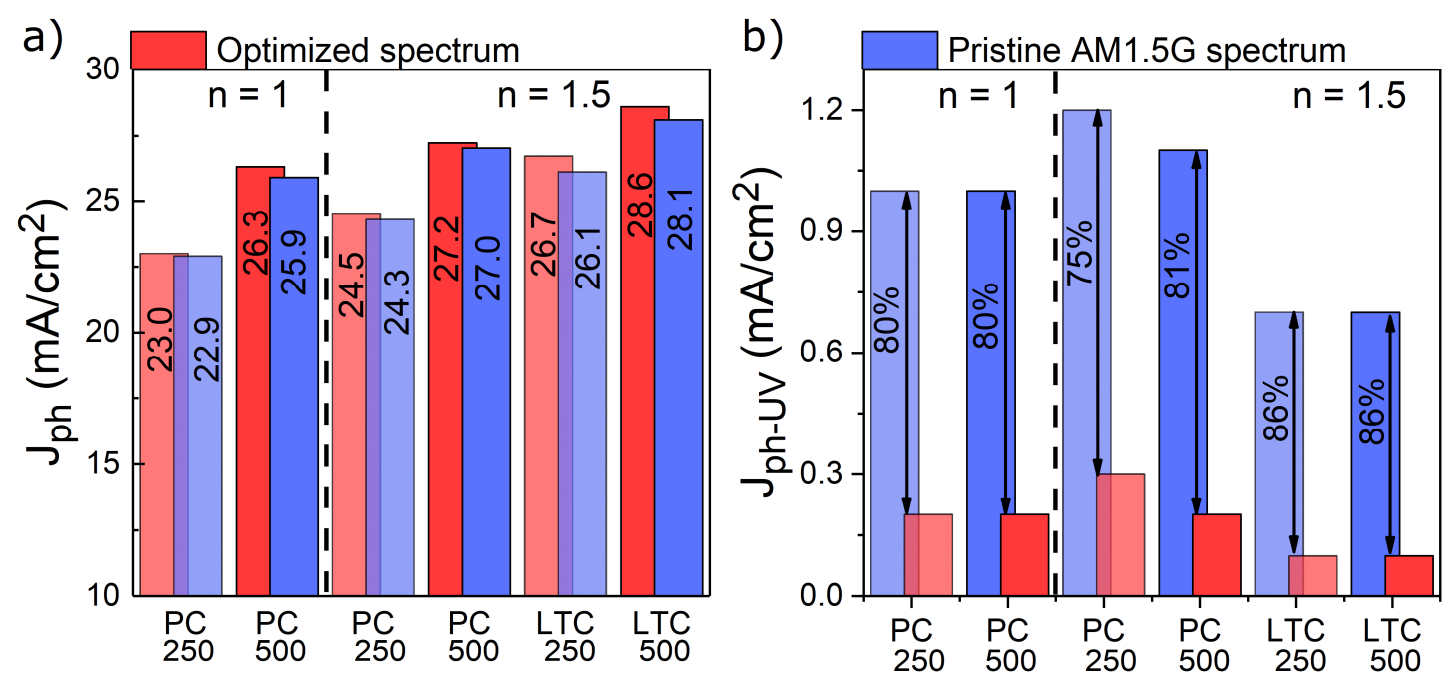

Figure 4 - Bar charts summarizing the results from the photocurrent sweeps attained with the pristine (blue) and optimized (red) spectra incident on the PC and LTC PSCs with either 250 or $500 \mathrm{~nm}$ Perovskite thickness. a) J $\mathrm{J}_{\text {ph }}$ values obtained considering the full UV-Visible-NIR wavelength range $(300-1000 \mathrm{~nm}) ;$ b) UV photocurrent $\left(J_{\text {ph-UV }}\right)$ values for wavelengths ranging from 300 to $400 \mathrm{~nm}$. The more transparent bars refer to the devices with $250 \mathrm{~nm}$ perovskite thickness, while the others refer to those with $500 \mathrm{~nm}$ perovskite thickness.

\section{Conclusion}

In conclusion, the use of optimized DS materials in the PSCs' encapsulation revealed a marginal increase in photocurrent at best of $2 \%\left(\sim 0.6 \mathrm{~mA} / \mathrm{cm}^{2}\right)$. This is due to the inevitable fact that there is not so much current that can be gained from exploiting the UV, and one is limited to the maximum gain that can be obtained by absorbing all this radiation, which of only $1.4 \mathrm{~mA} / \mathrm{cm}^{2}$. On the other hand, the use of unoptimized $\Delta \lambda$ and $\lambda_{C}$ revealed a severe impact in the optical performance of the cells, reducing the photocurrent by $7 \%$ and $12 \%$ for the worst cases simulated of the LTCs and PCs, respectively. The optimum $\Delta \lambda$ and $\lambda_{C}$ implied a spectrum shift to wavelengths around $500 \mathrm{~nm}$, matching well with the PSC's electrical performance peak, that could imply an increased device's efficiency surpassing the gains determined in this work. 
Importantly, from the analysis of the perovskite stability, one obtained a significant reduction in the $\mathrm{TiO}_{2}$ harmful photo-generation of one order of magnitude, coupled with an increase in the perovskite's bulk generation. The LTCs revealed a further decrease in the $\mathrm{TiO}_{2}$ photo-generation near the perovskite/TiO $\mathrm{Ti}_{2}$ interface due to the UV shading effect provided by the LT structures. By assessing the perovskite UV photocurrent for the different simulated cells, reductions up to $86 \%$ were obtained when comparing $\mathrm{J}_{\mathrm{ph}}$ values for the pristine and changed spectrum. Therefore, from these analyses, one can infer that the use of LDS avoids the unwanted effects of UV radiation on the perovskite, demonstrated by the hefty decrease in UV absorption coupled with the diminished $\mathrm{TiO}_{2}$ photoactivity resulting from lower photogeneration.

\section{Acknowledgments}

This work was funded by FEDER funds, through the COMPETE 2020 Program, and national funds, through the Portuguese Foundation for Science and Technology (FCTMEC), under the projects POCI-01-0145-FEDER-007688 (Reference UID/CTM/50025), ALTALUZ (Reference PTDC/CTM-ENE/5125/2014), SuperSolar (PTDC/NAN-OPT/28430/2017) and TACIT (PTDC/NAN-OPT/28837/2017). The authors also acknowledge funding from the European Project APOLO (H2020-LCE2017-RES-RIA, grant 763989). M. J. Mendes and S. Haque acknowledge funding by FCT-MEC through the grants SFRH/BPD/115566/2016 and PD/BD/143031/2018 (via AdvaMTech PhD program), respectively.

\section{Supplementary Information}

The details of the material's properties used for the simulations, and additional complementary figures are provided in supplementary information.

\section{Author Contributions}

M.A conceived and executed the simulation methodology, analyzed the results and wrote the manuscript. M.C. assisted in developing the simulation methodology and results analysis. S.H. assisted in the figures' composition and provided the parameters for the solar cells' simulation. M.J.M. projected and supervised the theoretical investigations presented here and assisted in the simulation methodology and results analysis. H.A. assisted in supervising the work, discussed the results, and revised the manuscript. E.F. and R.M. revised the manuscript and supervised the work and financing projects. 


\section{References}

(1) SolarPower Europe. Global Market Outlook for Solar Power 2017-2021. Solarpower Europe 2017.

(2) Philipps, S.; Warmuth, W. CFraunhofer ISE: Photovoltaics Report. 2018, p 45.

(3) International Energy Agency. Snapshot of Global Photovoltaic Markets. 2017.

(4) Green, M. A. Commercial Progress and Challenges for Photovoltaics. Nat. Energy 2016, 1 (1), 15015. https://doi.org/10.1038/nenergy.2015.15.

(5) Brongersma, M. L.; Cui, Y.; Fan, S. Light Management for Photovoltaics Using High-Index Nanostructures. Nat. Mater. 2014, 13 (5), 451-460. https://doi.org/10.1038/nmat3921.

(6) Isabella, O.; Vismara, R.; Linssen, D. N. P.; Wang, K. X.; Fan, S.; Zeman, M. Advanced Light Trapping Scheme in Decoupled Front and Rear Textured ThinFilm Silicon Solar Cells. Sol. Energy 2018, 162 (October 2017), 344-356. https://doi.org/10.1016/j.solener.2018.01.040.

(7) Mendes, M. J.; Haque, S.; Sanchez-Sobrado, O.; Araújo, A.; Águas, H.; Fortunato, E.; Martins, R. Optimal-Enhanced Solar Cell Ultra-Thinning with Broadband Nanophotonic Light Capture. iScience 2018, 3, 238-254. https://doi.org/10.1016/j.isci.2018.04.018.

(8) T. Vicente, A.; Araújo, A.; Mendes, M. J.; Nunes, D.; Oliveira, M. J.; SanchezSobrado, O.; Ferreira, M. P.; Águas, H.; Fortunato, E.; Martins, R. Multifunctional Cellulose-Paper for Light Harvesting and Smart Sensing Applications. J. Mater. Chem. C 2018, 6 (13), 3143-3181. https://doi.org/10.1039/C7TC05271E.

(9) Correa-Baena, J.-P.; Saliba, M.; Buonassisi, T.; Grätzel, M.; Abate, A.; Tress, W.; Hagfeldt, A. Promises and Challenges of Perovskite Solar Cells. Science (80-. 2017, 358 (6364), 739-744. https://doi.org/10.1126/science.aam6323.

(10) Filip, M. R.; Verdi, C.; Giustino, F. GW Band Structures and Carrier Effective Masses of $\mathrm{CH}_{3} \mathrm{NH}_{3} \mathrm{PbI}_{3}$ and Hypothetical Perovskites of the Type $\mathrm{APbI}_{3}: \mathrm{A}=$ $\mathrm{NH}_{4}, \mathrm{PH}_{4}, \mathrm{AsH}_{4}$, and $\mathrm{SbH}_{4}$. J. Phys. Chem. C 2015, 119 (45), 25209-25219. https://doi.org/10.1021/acs.jpcc.5b07891.

(11) Yin, W.-J.; Yang, J.-H.; Kang, J.; Yan, Y.; Wei, S.-H. Halide Perovskite Materials for Solar Cells: A Theoretical Review. J. Mater. Chem. A 2015, 3 (17), 8926-8942. https://doi.org/10.1039/C4TA05033A.

(12) Brenner, T. M.; Egger, D. A.; Kronik, L.; Hodes, G.; Cahen, D. Hybrid Organicinorganic Perovskites: Low-Cost Semiconductors with Intriguing ChargeTransport Properties. Nat. Rev. Mater. 2016, 1 (1), 15007. https://doi.org/10.1038/natrevmats.2015.7. 
(13) Jeon, N. J.; Noh, J. H.; Yang, W. S.; Kim, Y. C.; Ryu, S.; Seo, J.; Seok, S. Il. Compositional Engineering of Perovskite Materials for High-Performance Solar Cells. Nature 2015, $517 \quad$ (7535), 476-480. https://doi.org/10.1038/nature14133.

(14) Phillips, L. J.; Rashed, A. M.; Treharne, R. E.; Kay, J.; Yates, P.; Mitrovic, I. Z.; Weerakkody, A.; Hall, S.; Durose, K. Dispersion Relation Data for Methylammonium Lead Triiodide Perovskite Deposited on a (100) Silicon Wafer Using a Two-Step Vapour-Phase Reaction Process. Data Br. 2015, 5, 926-928. https://doi.org/10.1016/j.dib.2015.10.026.

(15) Kojima, A.; Teshima, K.; Shirai, Y.; Miyasaka, T. Organometal Halide Perovskites as Visible-Light Sensitizers for Photovoltaic Cells. J. Am. Chem. Soc. 2009, 131 (17), 6050-6051. https://doi.org/10.1021/ja809598r.

(16) Fu, F.; Feurer, T.; Weiss, T. P.; Pisoni, S.; Avancini, E.; Andres, C.; Buecheler, S.; Tiwari, A. N. High-Efficiency Inverted Semi-Transparent Planar Perovskite Solar Cells in Substrate Configuration. Nat. Energy 2017, 2 (1), 16190. https://doi.org/10.1038/nenergy.2016.190.

(17) Yang, W. S.; Noh, J. H.; Jeon, N. J.; Kim, Y. C.; Ryu, S.; Seo, J.; Seok, S. I. High-Performance Photovoltaic Perovskite Layers Fabricated through Intramolecular Exchange. Science (80-. ). 2015, 348 (6240), 1234-1237. https://doi.org/10.1126/science.aaa9272.

(18) Asghar, M. I.; Zhang, J.; Wang, H.; Lund, P. D. Device Stability of Perovskite Solar Cells - A Review. Renew. Sustain. Energy Rev. 2017, 77 (July 2016), 131-146. https://doi.org/10.1016/j.rser.2017.04.003.

(19) Lee, S.-W.; Kim, S.; Bae, S.; Cho, K.; Chung, T.; Mundt, L. E.; Lee, S.; Park, S.; Park, H.; Schubert, M. C.; et al. UV Degradation and Recovery of Perovskite Solar Cells. Sci. Rep. 2016, 6 (1), 38150. https://doi.org/10.1038/srep38150.

(20) Ito, S.; Tanaka, S.; Manabe, K.; Nishino, H. Effects of Surface Blocking Layer of $\mathrm{Sb} 2 \mathrm{~S} 3$ on Nanocrystalline $\mathrm{TiO}_{2}$ for $\mathrm{CH}_{3} \mathrm{NH}_{3} \mathrm{PbI}_{3}$ Perovskite Solar Cells. J. Phys. Chem. C 2014, 118 (30), 16995-17000. https://doi.org/10.1021/jp500449z.

(21) Farooq, A.; Hossain, I. M.; Moghadamzadeh, S.; Schwenzer, J. A.; Abzieher, T.; Richards, B. S.; Klampaftis, E.; Paetzold, U. W. Spectral Dependence of Degradation under Ultraviolet Light in Perovskite Solar Cells. ACS Appl. Mater. Interfaces 2018, $10 \quad$ (26), 21985-21990. https://doi.org/10.1021/acsami.8b03024.

(22) Leijtens, T.; Eperon, G. E.; Pathak, S.; Abate, A.; Lee, M. M.; Snaith, H. J. Overcoming Ultraviolet Light Instability of Sensitized $\mathrm{TiO}_{2}$ with MesoSuperstructured Organometal Tri-Halide Perovskite Solar Cells. Nat. Commun. 
2013, 4, 1-8. https://doi.org/10.1038/ncomms3885.

(23) Quitsch, W.-A.; DeQuilettes, D. W.; Pfingsten, O.; Schmitz, A.; Ognjanovic, S.; Jariwala, S.; Koch, S.; Winterer, M.; Ginger, D. S.; Bacher, G. The Role of Excitation Energy in Photobrightening and Photodegradation of Halide Perovskite Thin Films. J. Phys. Chem. Lett. 2018, 9 (8), 2062-2069. https://doi.org/10.1021/acs.jpclett.8b00212.

(24) Gheno, A.; Trigaud, T.; Bouclé, J.; Audebert, P.; Ratier, B.; Vedraine, S. Stability Assessments on Luminescent Down-Shifting Molecules for UVProtection of Perovskite Solar Cells. Opt. Mater. (Amst). 2018, 75, 781-786. https://doi.org/10.1016/j.optmat.2017.11.027.

(25) Anizelli, H. S.; Stoichkov, V.; Fernandes, R. V.; Duarte, J. L.; Laureto, E.; Kettle, J.; Visoly-Fisher, I.; Katz, E. A. Application of Luminescence Downshifting Materials for Enhanced Stability of $\mathrm{CH}_{3} \mathrm{NH}_{3} \mathrm{PbI}_{3(1-x)} \mathrm{Cl}_{3 x}$ Perovskite Photovoltaic Devices. Org. Electron. 2017, 49, 129-134. https://doi.org/10.1016/j.orgel.2017.06.056.

(26) Chander, N.; Khan, A. F.; Chandrasekhar, P. S.; Thouti, E.; Swami, S. K.; Dutta, V.; Komarala, V. K. Reduced Ultraviolet Light Induced Degradation and Enhanced Light Harvesting Using $\mathrm{YVO}_{4}: \mathrm{Eu}_{3}{ }^{+}$down-Shifting Nano-Phosphor Layer in Organometal Halide Perovskite Solar Cells. Appl. Phys. Lett. 2014, 105 (3), 033904. https://doi.org/10.1063/1.4891181.

(27) Klampaftis, E.; Ross, D.; McIntosh, K. R.; Richards, B. S. Enhancing the Performance of Solar Cells via Luminescent Down-Shifting of the Incident Spectrum: A Review. Sol. Energy Mater. Sol. Cells 2009, 93 (8), 1182-1194. https://doi.org/10.1016/j.solmat.2009.02.020.

(28) Liu, N.; Xue, H.; Ji, Y.; Wang, J. ZnSe/ZnS Core-Shell Quantum Dots Incorporated with Ag Nanoparticles as Luminescent down-Shifting Layers to Enhance the Efficiency of Si Solar Cells. J. Alloys Compd. 2018, 747, 696-702. https://doi.org/10.1016/j.jallcom.2018.03.060.

(29) Lesyuk, R.; Lesnyak, V.; Herguth, A.; Popovych, D.; Bobitski, Y.; Klinke, C.; Gaponik, N. Simulation Study of Environmentally Friendly Quantum-Dot-Based Photovoltaic Windows. J. Mater. Chem. C 2017, 5 (45), 11790-11797. https://doi.org/10.1039/C7TC02945D.

(30) Lesyuk, R.; Marinov, V.; Hobbie, E. K.; Elbaradei, A.; Tarnavchyk, I.; Bobitski, Y. Toward Cadmium-Free Spectral down-Shifting Converters for Photovoltaic Applications. Sol. Energy Mater. Sol. Cells 2016, 151, 52-59. https://doi.org/10.1016/j.solmat.2016.02.021.

(31) Chatten, A. J.; Barnham, K. W. J.; Buxton, B. F.; Ekins-Daukes, N. J.; Malik, M. A. A New Approach to Modelling Quantum Dot Concentrators. Sol. Energy 
Mater. Sol. Cells 2003, 75 (3-4), 363-371. https://doi.org/10.1016/S09270248(02)00182-4.

(32) Chatten, A. J.; Barnham, K. W. J.; Buxton, B. F.; Ekins-Daukes, N. J.; Malik, M. A. Quantum Dot Solar Concentrators. Semiconductors 2004, 38 (8), 909917. https://doi.org/10.1134/1.1787111.

(33) Rondão, R.; Frias, A. R.; Correia, S. F. H.; Fu, L.; de Zea Bermudez, V.; André, P. S.; Ferreira, R. A. S.; Carlos, L. D. High-Performance Near-Infrared Luminescent Solar Concentrators. ACS Appl. Mater. Interfaces 2017, 9 (14), 12540-12546. https://doi.org/10.1021/acsami.7b02700.

(34) Lipovšek, B.; Solodovnyk, A.; Forberich, K.; Stern, E.; Krč, J.; Brabec, C. J.; Topič, M. Optical Model for Simulation and Optimization of Luminescent DownShifting Layers Filled with Phosphor Particles for Photovoltaics. Opt. Express 2015, 23 (15), A882. https://doi.org/10.1364/OE.23.00A882.

(35) McIntosh, K. R.; Lau, G.; Cotsell, J. N.; Hanton, K.; Bätzner, D. L.; Bettiol, F.; Richards, B. S. Increase in External Quantum Efficiency of Encapsulated Silicon Solar Cells from a Luminescent Down-Shifting Layer. Prog. Photovoltaics Res. Appl. 2009, 17 (3), 191-197. https://doi.org/10.1002/pip.867.

(36) Correia, S. F. H.; Lima, P. P.; Pecorato, E.; Ribeiro, S. J. L.; André, P. S.; Ferreira, R. A. S.; Carlos, L. D. Scale up the Collection Rea of Luminescent Solar Concentrators towards Metre-Length Flexible Waveguiding Photovoltaics. Prog. Photovoltaics Res. Appl. 2016, 24, 1178-1193. https://doi.org/10.1002/pip.

(37) Correia, S. F. H.; Lima, P. P.; André, P. S.; Ferreira, M. R. S.; Carlos, L. A. D. High-Efficiency Luminescent Solar Concentrators for Flexible Waveguiding Photovoltaics. Sol. Energy Mater. Sol. Cells 2015, 138, 51-57. https://doi.org/10.1016/j.solmat.2015.02.032.

(38) Wiegman, J. W. E.; van der Kolk, E. Building Integrated Thin Film Luminescent Solar Concentrators: Detailed Efficiency Characterization and Light Transport Modelling. Sol. Energy Mater. Sol. Cells 2012, 103, 41-47. https://doi.org/10.1016/j.solmat.2012.04.016.

(39) Kettle, J.; Bristow, N.; Gethin, D. T.; Tehrani, Z.; Moudam, O.; Li, B.; Katz, E. A.; dos Reis Benatto, G. A.; Krebs, F. C. Printable Luminescent down Shifter for Enhancing Efficiency and Stability of Organic Photovoltaics. Sol. Energy Mater. Sol. Cells 2016, 144, 481-487. https://doi.org/10.1016/j.solmat.2015.09.037.

(40) Llanos, J.; Brito, I.; Espinoza, D.; Sekar, R.; Manidurai, P. A Down-Shifting $\mathrm{Eu}_{3}{ }^{+}$-Doped $\mathrm{Y}_{2} \mathrm{WO}_{6} / \mathrm{TiO}_{2}$ Photoelectrode for Improved Light Harvesting in DyeSensitized Solar Cells. R. Soc. Open Sci. 2018, 5 (2), 171054. 
https://doi.org/10.1098/rsos.171054.

(41) Yin, G.; Manley, P.; Schmid, M. Light Trapping in Ultrathin CuIn ${ }_{1-x} \mathrm{Ga}_{x} \mathrm{Se}_{2}$ Solar Cells by Dielectric Nanoparticles. Sol. Energy 2018, 163 (January), 443-452. https://doi.org/10.1016/j.solener.2018.01.096.

(42) Baranov, D. G.; Zuev, D. A.; Lepeshov, S. I.; Kotov, O. V.; Krasnok, A. E.; Evlyukhin, A. B.; Chichkov, B. N. All-Dielectric Nanophotonics: The Quest for Better Materials and Fabrication Techniques. 2017, 4 (7). https://doi.org/10.1364/OPTICA.4.000814.

(43) Bhattacharya, J.; Chakravarty, N.; Pattnaik, S.; Dennis Slafer, W.; Biswas, R.; Dalal, V. L. A Photonic-Plasmonic Structure for Enhancing Light Absorption in Thin Film Solar Cells. Appl. Phys. Lett. 2011, 99 (13), 131114. https://doi.org/10.1063/1.3641469.

(44) Xiao, J.; Fang, H.; Su, R.; Li, K.; Song, J.; Krauss, T. F.; Li, J.; Martins, E. R. Paths to Light Trapping in Thin Film GaAs Solar Cells. Opt. Express 2018, 26 (6), A341. https://doi.org/10.1364/OE.26.00A341.

(45) Donie, Y. J.; Smeets, M.; Egel, A.; Lentz, F.; Preinfalk, J. B.; Mertens, A.; Smirnov, V.; Lemmer, U.; Bittkau, K.; Gomard, G. Light Trapping in Thin Film Silicon Solar Cells via Phase Separated Disordered Nanopillars. Nanoscale 2018, 10 (14), 6651-6659. https://doi.org/10.1039/C8NR00455B.

(46) Sanchez-Sobrado, O.; Mendes, M. J.; Haque, S.; Mateus, T.; Araujo, A.; Aguas, H.; Fortunato, E.; Martins, R. Colloidal-Lithographed $\mathrm{TiO}_{2}$ Photonic Nanostructures for Solar Cell Light Trapping. J. Mater. Chem. C 2017, 5 (27), 6852-6861. https://doi.org/10.1039/C7TC01756A.

(47) Grandidier, J.; Deceglie, M. G.; Callahan, D. M.; Atwater, H. A. Simulations of Solar Cell Absorption Enhancement Using Resonant Modes of a Nanosphere Array. In Physics, Simulation, and Photonic Engineering of Photovoltaic Devides; Freundlich, A., Guillemoles, J.-F. F., Eds.; 2012; Vol. 8256, p 825603. https://doi.org/10.1117/12.909677.

(48) Deceglie, M. G.; Ferry, V. E.; Alivisatos, A. P.; Atwater, H. A. Design of Nanostructured Solar Cells Using Coupled Optical and Electrical Modeling. Nano Lett. 2012, 12 (6), 2894-2900. https://doi.org/10.1021/nl300483y.

(49) Haque, S.; Mendes, M. J. de M. D.; Sanchez-Sobrado, O.; Águas, H.; Fortunato, E.; Martins, R. Photonic-Structured $\mathrm{TiO}_{2}$ for High-Efficiency, Flexible and Stable Perovskite Solar Cells. Nano Energy 2019, 59 (January), 91-101. https://doi.org/10.1016/j.nanoen.2019.02.023.

(50) Uekert, T.; Solodovnyk, A.; Ponomarenko, S.; Osvet, A.; Levchuk, I.; Gast, J.; Batentschuk, M.; Forberich, K.; Stern, E.; Egelhaaf, H.-J.; et al. Nanostructured Organosilicon Luminophores in Highly Efficient Luminescent 
Down-Shifting Layers for Thin Film Photovoltaics. Sol. Energy Mater. Sol. Cells 2016, 155, 1-8. https://doi.org/10.1016/j.solmat.2016.04.019.

(51) Lumerical Inc. https://www.lumerical.com/, Last accessed on: March 2019.

(52) Purcell-Milton, F.; Gun'ko, Y. K. Quantum Dots for Luminescent Solar Concentrators. J. Mater. Chem. 2012, 22 (33), 16687. https://doi.org/10.1039/c2jm32366d.

(53) Wang, B.; Li, B.; Shen, T.; Li, M.; Tian, J. ZnSe Quantum Dots Downshifting Layer for Perovskite Solar Cells. J. Energy Chem. 2018, 27 (3), 736-741. https://doi.org/10.1016/j.jechem.2017.11.021.

(54) Richards, B. S.; McIntosh, K. R. Overcoming the Poor Short Wavelength Spectral Response of CdS/CdTe Photovoltaic Modules via Luminescence downShifting: Ray-Tracing Simulations. Prog. Photovoltaics Res. Appl. 2007, 15 (1), 27-34. https://doi.org/10.1002/pip.723.

(55) National Energy Renewable Laboratory (NREL) https://www.nrel.gov/, Last accessed on: March 2019.

(56) Hou, X.; Xuan, T.; Sun, H.; Chen, X.; Li, H.; Pan, L. High-Performance Perovskite Solar Cells by Incorporating a $\mathrm{ZnGa}_{2} \mathrm{O}_{4}: \mathrm{Eu}_{3}{ }^{+}$Nanophosphor in the Mesoporous $\mathrm{TiO}_{2}$ Layer. Sol. Energy Mater. Sol. Cells 2016, 149, 121-127. https://doi.org/10.1016/j.solmat.2016.01.021.

(57) Yang, Y.; Yang, M.; Moore, D. T.; Yan, Y.; Miller, E. M.; Zhu, K.; Beard, M. C. Top and Bottom Surfaces Limit Carrier Lifetime in Lead Iodide Perovskite Films. Nat. Energy 2017, 2 (2), 16207. https://doi.org/10.1038/nenergy.2016.207. 